\title{
Encapsulating Peritoneal Sclerosis Presenting after Two Donor Kidney Transplantations: A Case Report and Literature Review
}

\author{
Sasmit Roy ${ }^{a}$ Kyrstin Alexander ${ }^{b} \quad$ Monzurul Hasan Chowdhury ${ }^{c}$ \\ Vijay Vangurid $^{d}$ Kenneth Bodziak ${ }^{b}$ \\ aDivision of Nephrology, University of Virginia, Charlottesville, VA, USA; ${ }^{b}$ Division of Renal \\ Medicine, University of Massachusetts Medical School, Worcester, MA, USA; IInternal \\ Medicine, Frank H Netter MD School of Medicine, North Haven, CT, USA; ${ }^{d}$ Division of \\ Pathology, University of Massachusetts Medical School, Worcester, MA, USA
}

\section{Keywords}

Encapsulating peritoneal sclerosis - Kidney transplantation - Peritoneal dialysis - Tamoxifen . End-stage renal disease

\begin{abstract}
Encapsulating peritoneal sclerosis is a rare, recurring complication in peritoneal dialysis (PD) patients. With a mortality rate of $51 \%$, it continues to be a therapeutic enigma among clinicians. However, the incidence after kidney transplantation (KT) has rarely been reported. We report a unique case of encapsulating peritoneal sclerosis (EPS), occurring years after failed initial living KT, and diagnosed after second deceased donor kidney transplantation. A 35-yearold male, on prior PD for 4 years, followed by failed KT of 8 years, was presented with abdominal pain, weight loss, and vomiting, 7 months after his second deceased donor KT. An abdominal computed tomography showed intra-abdominal loculated fluid collection, but no obstruction. Exploratory laparotomy revealed extensive peritoneal thickening and blocked intestinal loops. Histopathology was indicative of EPS with fibrous adhesions and sclerotic tissues. Besides restarting his immunosuppressive medications, tamoxifen therapy was initiated as definitive medical management. Currently, he is in clinical remission, follows at transplant clinic, and still experiences episodes of small bowel obstruction. Though the incidence of EPS after KT has been observed sporadically worldwide, none has been reported in the USA. Despite its prevalence in PD patients, therapeutic interventions attempted so far, are not definitive.

(C) 2021 The Author(s).

Published by S. Karger AG, Basel
\end{abstract}




\section{Introduction}

Encapsulating peritoneal sclerosis (EPS) is a rare, dreadful, and recurrent complication in patients undertaking peritoneal dialysis (PD). Although prevalent since 1907, this disease is still a diagnostic and management challenge among practicing clinicians everywhere. It entails a mortality rate of $51 \%$, in the initial year, and has an incidence of $0.7-3.3 \%$ [1], which might be up to 13.6 per 1,000 patient-years [2].

The 2 most commonly implicated triggers in PD patients include prior history of peritonitis and extended duration of PD. This is succeeded by a second insult like peritonitis, surgery, hemoperitoneum on the damaged peritoneum [3]. Only a few cases of EPS have been reported in kidney transplantation (KT) recipients [4-6], but none in North America. Our case highlights a unique presentation of EPS, diagnosed years after failed living KT, and presenting after his second deceased donor KT.

\section{Case Report}

A 35-year-old African-American male presented to the emergency department, with chief complaints of nausea, vomiting, and abdominal distension for 2 months. He had persistent odynophagia with loss of appetite and profound weight loss. His notable past medical history included end-stage renal disease, from biopsy-proven focal segmental glomerular sclerosis; prior living KT 10 years ago (from mother), which failed after 8 years due to medicine noncompliance and recurrent focal segmental glomerular sclerosis. He was on PD for 4 years before his initial KT. Post first KT failure, he was on hemodialysis for 2 years before he received his second KT from a deceased donor. During his second transplantation, the hospital course was associated with complications like delayed graft function recovery, deep vein thrombosis. His transplant induction medications were rabbit antithymocyte globulin and corticosteroids. His maintenance immunosuppressant regimen being tablets tacrolimus $4 \mathrm{mg}$ twice daily, mycophenolate mofetil $250 \mathrm{mg}$ twice daily and prednisone 5 mg daily.

On physical examination, his blood pressure was $140 / 84 \mathrm{~mm} \mathrm{Hg}$, pulse rate $89 / \mathrm{min}$, saturating $99 \%$ on room air, and his temperature was normal. He was severely cachexia and had oral thrush. Gastrointestinal (GI) system examination findings included abdominal distension, with diffuse tenderness and diminished bowel sounds. The rest of the systemic findings including respiratory, cardiovascular, and neurological systems were unremarkable. Pertinent lab findings were serum creatinine $2 \mathrm{mg} / \mathrm{dL}$ ([reference 0.6-1.3 mg/dL], which was his baseline), blood urea nitrogen $54 \mathrm{mg} / \mathrm{dL}$ (reference $8-20 \mathrm{mg} / \mathrm{dL}$ ). Admission hematocrit was 33\%. On hospitalization day 2, he experienced left upper quadrant acute sharp abdominal pain, 8/10 in intensity, nonradiating, with drop-in hematocrit from 33 to $23 \%$, his condition deteriorated with blood pressure dropping to $70 / 40 \mathrm{~mm} \mathrm{Hg}$ and requiring vasopressor support. Computerized tomography of the abdomen demonstrated loculated intra-abdominal fluid collections, shown in Figure 1. Diagnostic paracentesis revealed no signs of infection. Stool guaiac returned negative. The patient was treated conservatively with the cessation of warfarin and packed red blood cell transfusion. A bone marrow biopsy was done for suspected post-transplant lymphoproliferative disorder which returned negative. He was started on total parental nutrition for his persistent cachectic state. Suspecting EPS, an exploratory laparotomy was undertaken. This revealed extensive peritoneal thickening with signs of chronic inflammation along with adhesions and blocked intestinal loops, as shown in Figure 2. His abdomen was thoroughly washed and adhesiolysis was done. He was thereafter started on oral calcineurin

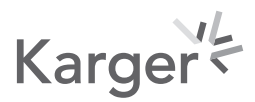


Fig. 1. Computed tomogram abdomen showing loculated intra-abdominal fluid collections.

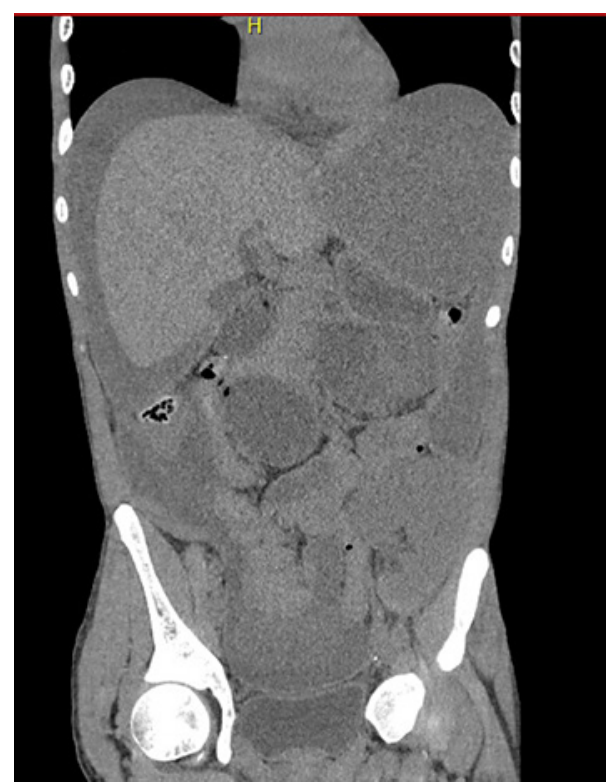

Fig. 2. Intraoperative findings of extensive peritoneal thickening with signs of chronic inflammation.

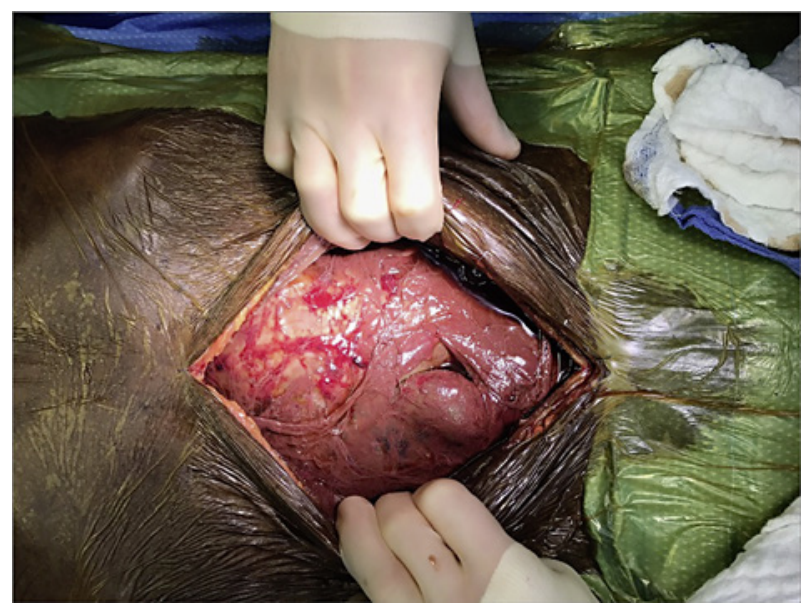

inhibitor, that is, tamoxifen therapy, $10 \mathrm{mg}$ twice daily. He continues to be on the same dose at present. Histopathology results returned positive for EPS findings, with encapsulating fibrous adhesions over terminal ileum and sclerotic tissues over intestinal wall smooth muscles as shown in Figure 3. The patient was subsequently able to tolerate oral intake, and he was successfully weaned off total parental nutrition. He was gradually initiated back on his immunosuppressants and discharged in stable condition. Throughout this hospitalization, his serum creatinine stayed stable at around 2-2.2 mg/dL.

He follows with the renal transplant clinic and continues to be on oral tamoxifen $10 \mathrm{mg}$ twice daily, and other oral immunosuppressants, namely tacrolimus $4 \mathrm{mg}$ twice daily, mycophenolate mofetil $250 \mathrm{mg}$ twice daily and prednisone $5 \mathrm{mg}$ daily. It has been 3 years since he has been last operated and he has been doing fairly well with occasional episodes of relapses. His abdominal symptoms recur periodically with small bowel obstructions. He has refused any further surgeries. Written consent was obtained from the patient before writing this case report. 
Fig. 3. Portion of encapsulating fibrous adhesions over terminal ileum. Smooth muscle from the intestinal wall (bottom) is covered by sclerotic tissue $\left({ }^{*}\right)$ with overlying fibrin deposition (top).

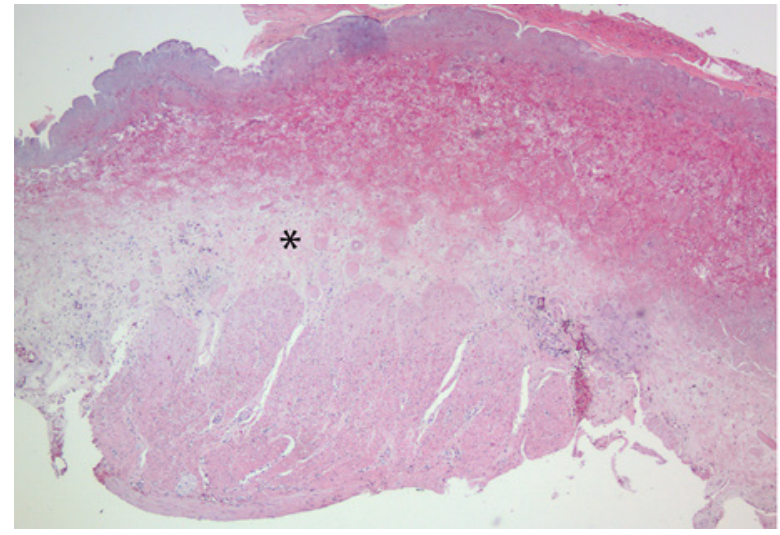

\section{Discussion}

PD is a successful modality of dialysis for end-stage renal disease patients which gives flexibility to them in managing their treatment, without compromising their quality of life. Overall, it is a very safe modality of dialysis. However, one of its dreadful complications, which are becoming more prevalent with wider use of PD, is encapsulating peritoneal sclerosis (EPS). EPS was initially observed in 1907 when it was coined as "Peritonitis Chronica Fibrosa Encapsulate." This encases a clinical and pathological entity with intestinal obstruction and microscopic sclerosing features. EPS is characterized by bowel loops getting encased from extensive intraperitoneal fibrosis [7].

EPS has an early inflammatory stage, succeeded by the sclerosing stage developing into an encapsulating cocoon, and thereby causing GI symptoms. EPS clinical features stem from underlying inflammation, peritoneal adhesions, and/or gut ileus. Initially, EPS mostly has benign symptoms like nausea, vomiting, progressive loss of appetite, weakness with intermittent periods of quiescence. It is typically associated with progressive ultrafiltration loss, causing edema and fluid retention. EPS is a gradual, insidious, nonacute clinical entity. Subsequent stages present as an abdominal mass, abdominal pain, ascites, and constipation [3]. These subtle symptoms and intermittent progression of EPS separate it from other GI disorders frequently encountered, for example, gut perforation, bowel obstruction, etc. [4].

Overall, the cause of EPS is believed to be multifactorial and no specific pathology has been identified. Most studies have agreed on prolonged PD duration as a risk factor [7-9].

Various other parameters implicated in worldwide studies are - young individuals, higher dialysate glucose concentration, peritonitis (prolonged/severe), using conventional PD solutions (unlike the newer biocompatible PD solutions), icodextrin use, abdominal operative procedures, $\beta$-blockers use, KT (as our case), ultrafiltration failure, and higher peritoneal solute transport rate. However, these data are scattered and not completely implicit [10]. Calcineurin inhibitors, for example, cyclosporine, tacrolimus, which potentiates the transforming growth factor $\beta$ expression, thereby leading to progressive peritoneal fibrosis, can be a possible reason in KT recipients [11].

The preferred diagnostic imaging modality is an abdominal computerized tomography scan. Standalone imaging features of EPS are bowel thickening, peritoneal calcification, bowel dilatation, and bowel tethering [3]. Occasionally, laparotomy and/or laparoscopic diagnostic confirmation is necessary. The inherent surgical features include peritoneal thickening enclosing intestinal contents [12].

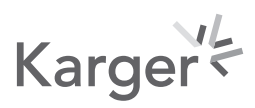


Histologically, EPS is identified by significant peritoneal membrane thickening with dense layers of fibro-connective tissue, and loss of mesothelium; exudates, ossification, increase in fibrin, calcification, and interstitial fibrosis are other notable features [3].

Although not fruitful always, the management consensus of EPS has historically included the cessation of PD with the transition to hemodialysis, bowel rest with parenteral nutrition, and therapeutic interventions like immunosuppressive medications and/or surgery [10].

Successful therapeutic medical interventions reported in EPS literature include tamoxifen [13], corticosteroids [14], and immunosuppression [15]. Most of these treatment modalities are in individual case series or small studies. Previously, the prognosis with surgical therapy was extremely poor. More recent studies done in Japan, UK, and Germany have shown encouraging surgical results [10]. Surgical treatment is highly complex because the pathology of EPS includes adhesive processes throughout the small intestine, and the end goal of the procedure is to release these adhesions from the intestine.

Transplantation is prophesied to cause a survival benefit among EPS individuals. Few retrospective studies have predicted better survival among them when they undergo KT [13].

Our case tries to highlight the rare risk factor of KT culminating into this scarce, recurring complication of EPS in PD patients. While studies have suggested KT may benefit EPS, we try to highlight that KT may increase the likelihood of this serious complication. Though few cases have been reported in post-KT recipients [4-6] - to our knowledge, this could be the first case reported from North America, where EPS has been diagnosed after second KT.

\section{Conclusion}

EPS is a rare but dreadful complication of PD which can happen in individuals on the extended duration of PD. While only a few cases have been reported, the incidence of EPS following KT, that too after second transplantation, is extremely rare. Our case could be the first reported case of EPS in North America, in an individual who presented after 2 successive KTs. Also, tamoxifen therapy is a fairly well-tested option with limited efficacy. Clinicians should be mindful of this rare condition presenting as a diagnostic and management challenge to the medical fraternity. The fact that it can occur as a complication of KT even after suspending PD for many years should be a teaching point for every nephrologist. More research needs to be done to help treat these hapless patients better.

\section{Statement of Ethics}

Written informed consent was obtained from the patient for publication of this case report and any accompanying images.

\section{Conflicts of Interest Statement}

The authors of this manuscript have no conflicts of interest to declare.

\section{Funding Sources}

The authors did not receive any funding.

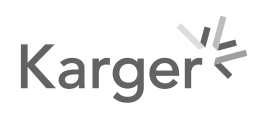




\section{Author Contributions}

Sasmit Roy: case writing, corresponding author, and direct patient care; Kyrstin Alexander; Monzurul Hasan Chowdhury; Kenneth Bodziak: literature search, case writing, and direct patient care; and Vijay Vanguri: pathology interpretation and pathology slides.

\section{References}

1 Brown MC, Simpson K, Kerssens JJ, Mactier RA; Scottish Renal Registry. Encapsulating peritoneal sclerosis in the new millennium: a national cohort study. Clin J Am Soc Nephrol. 2009;4(7):1222-9.

2 Burkart JM. Encapsulating peritoneal sclerosis in peritoneal dialysis patients. In: Post T, editor. UpToDate. Waltham, MA: UpToDate; 2020. Available from: www.uptodate.com Accessed 2020 May 14.

3 de Sousa E, del Peso-Gilsanz G, Bajo-Rubio MA, Ossorio-González M, Selgas-Gutiérrez R. Encapsulating peritoneal sclerosis in peritoneal dialysis. A review and European initiative for approaching a serious and rare disease. Nefrologia. 2012;32(32):707-14.

4 Nakamoto H. Encapsulating peritoneal sclerosis: a clinician's approach to diagnosis and medical treatment. Perit Dial Int. 2005;25(Suppl):S30-8.

5 Korte MR, Sampimon DE, Betjes MG, Krediet RT. Encapsulating peritoneal sclerosis: the state of affairs. Nat Rev Nephrol. 2011;7(7):528-38.

6 Caicedo L, Delgado A, Caicedo LA, Bravo JC. Sclerosing encapsulated peritonitis: a devastating and infrequent disease complicating kidney transplantation, case report and literature review. Int J Surg Case Rep. 2017;33: 135.

7 Brown EA, Van Biesen W, Finkelstein FO, Hurst H, Johnson DW, Kawanishi H, et al. Length of time on peritoneal dialysis and encapsulating peritoneal sclerosis: position paper for ISPD. Perit Dial Int. 2009;29(6):595-600.

8 Nakayama M, Miyazaki M, Honda K, Kasai K, Tomo T, Nakamoto H, et al. Encapsulating peritoneal sclerosis in the era of a multi-disciplinary approach based on biocompatible solutions: the NEXT-PD study. Perit Dial Int. 2014;34(7):766-74.

9 Vizzardi V, Sandrini M, Zecchini S, Ravera S, Manili L, Cancarini G. Encapsulating peritoneal sclerosis in an Italian center: thirty year experience. J Nephrol. 2016;29(29):259-67.

10 Brown EA, Bargman J, Van Biesen W, Chang MY, Finkelstein FO, Hurst H, et al. Length of time on peritoneal dialysis and encapsulating peritoneal sclerosis: position paper for ISPD: 2017 update. Perit Dial Int. 2017; 37(4):362-74.

11 Khanna A, Plummer M, Bromberek C, Bresnahan B, Hariharan S. Expression of TGF-beta and fibrogenic genes in transplant recipients with tacrolimus and cyclosporine nephrotoxicity. Kidney Int. 2002;62(6):2257-63.

12 Kawanishi H, Moriishi M, Tsuchiya S. Experience of 100 surgical cases of encapsulating peritoneal sclerosis: investigation of recurrent cases after surgery. Adv Perit Dial. 2006;22(22):60.

13 Mohamed AO, Kamar N, Nogier MB, Esposito L, Duffas JP, Rostaing L. Tamoxifen therapy in kidney-transplant patients presenting with severe encapsulating peritoneal sclerosis after treatment for acute humoral rejection. Exp Clin Transplant. 2009;7(3):164.

14 Kawanishi H, Kawaguchi Y, Fukui H, Hara S, Imada A, Kubo H, et al. Encapsulating peritoneal sclerosis in Japan: a prospective, controlled, multicenter study. Am J Kidney Dis. 2004;44(44):729-37.

15 Lafrance JP, Létourneau I, Ouimet D, Bonnardeaux A, Leblanc M, Mathieu N, et al. Successful treatment of encapsulating peritoneal sclerosis with immunosuppressive therapy. Am J Kidney Dis. 2008;51(51):e7-10. 